\title{
Amorphous B-doped graphitic carbon nitride quantum dots with high photoluminescence quantum yield of near $90 \%$ and their sensitive detection of $\mathrm{Fe}^{2+} / \mathrm{Cd}^{2+}$
}

\author{
Bo Li ${ }^{1}$, Jing Zhang ${ }^{2}$, Ziyu Luo ${ }^{1}$, Xinpei Duan ${ }^{3}$, Wei-Qing Huang ${ }^{1 *}$, Wangyu $\mathrm{Hu}^{{ }^{*}}$, Anlian Pan $^{1}$, \\ Lei Liao ${ }^{3}$, Lang Jiang ${ }^{2}$ and Gui-Fang Huang ${ }^{1^{*}}$
}

\begin{abstract}
Graphitic carbon nitride quantum dots (CNQDs) are emerging as attractive photoluminescent (PL) materials with excellent application potential in fluorescence imaging and heavy-metal ion detection. However, three limitations, namely, low quantum yields (QYs), self-quenching, and excitation-dependent PL emission behaviors, severely impede the commercial applications of crystalline CNQDs. Here we address these three challenges by synthesizing borondoped amorphous CNQDs via a hydrothermal process followed by the top-down cutting approach. Structural disorder endows the amorphous boron-doped CNQDs (B-CNQDs) with superior elastic strain performance over a wide range of $\mathrm{pH}$ values, thus effectively promoting mass transport and reducing exciton quenching. Boron as a dopant could fine-tune the electronic structure and emission properties of the PL material to achieve excitation-independent emission via the formation of uniform boron states. As a result, the amorphous B-CNQDs show unprecedented fluorescent stability (i.e., no obvious fading after two years) and a high QY of $87.4 \%$; these values indicate that the quantum dots obtained are very promising fluorescent materials. Moreover, the B-CNQDs show bright-blue fluorescence under ultraviolet excitation when applied as ink on commercially available paper and are capable of the selective and sensitive detection of $\mathrm{Fe}^{2+}$ and $\mathrm{Cd}^{2+}$ in the parts-per-billion range. This work presents a novel avenue and scientific insights on amorphous carbon-based fluorescent materials for photoelectrical devices and sensors.
\end{abstract}

Keywords: amorphous, boron doping, carbon nitride quantum dots, fluorescence imaging and sensors

\section{INTRODUCTION}

Fluorescence detection (FDT) is considered one of the most promising technologies for water quality evaluation, imaging, and biolabeling on account of its excellent sensitivity and operability [1-5]. As a key material for FDT, quantum dots (QDs) with a lateral scale of several nanometers have attracted great research attention [6-8]. The quantum confinement, surface state, and size effects of QDs give rise to intriguing properties in the resultant optical and electronic structures and conferring them with much broader applicability and higher fluorescence quantum yields (QYs) compared with traditional FDT materials [9-12]. Several QD-based FDT materials, such as CdS/CdTe [13-15], graphene [16-18], and carbon QDs, have recently been developed $[19,20]$. Unfortunately, the presence of heavy metals and potentially carcinogenic organic molecules in the majority of the available QDs could give rise to environmental problems and biological toxicity.

Graphitic carbon nitride QDs (CNQDs) are generally considered excellent candidates for fluorescence applications because of their low cost, high biocompatibility, low toxicity, and good water solubility [21-24]. Moreover, CNQDs consist of ordered tris-triazine moieties covalently connected by trigonal nitrogen and stacked in a graphitic fashion, which endows them with high QYs (e.g., 30\%-90\%) and tunable fluorescent properties $[25,26]$. This intrinsic characteristic of CNQDs distinguishes them from traditional fluorescent materials and indicates their prospective applications in metal ion detection [27], security ink [3], and optoelectronic devices

\footnotetext{
${ }^{1}$ College of Physics and Electronics and College of Materials Science and Engineering, Hunan University, Changsha 410082, China

${ }^{2}$ Institute of Chemistry, Chinese Academy of Sciences, Beijing 100049, China

${ }^{3}$ Key Laboratory for Micro/Nano Optoelectronic Devices of Ministry of Education \& Hunan Provincial Key Laboratory of Low-Dimensional Structural Physics and Devices, College of Physics and Electronics, Hunan University, Changsha 410082, China

* Corresponding authors (emails: wqhuang@hnu.edu.cn (Huang WQ); wyuhu@hnu.edu.cn (Hu W); gfhuang@hnu.edu.cn (Huang GF))
} 
[28-30]. Nevertheless, major efforts in the FDT field are so far emphasized on the crystalline CNQDs-based materials. Similar to other metal-free-based QDs, crystalline CNQD-based FDT materials also present three primary drawbacks (i.e., low QYs, self-quenching and excitationdependent properties), because of the formation of an intramolecular ground-state dimer complex or energy transfer between the adjacent QDs [31-33]. Fortunately, a growing class of amorphous QDs has been demonstrated to have more efficient FDT performance than their crystalline counterparts $[4,32,34]$. The isotropic characteristics of amorphous QDs induced by their shortrange structural ordering endow these dots with longterm operating stability over a wide range of $\mathrm{pH}$ values, provide open active diffusion channels that could facilitate ion transport, and contribute to the formation of excitons, resulting in improvements in photoluminescent (PL) QY. The rational design and synthesis of amorphous CNQDs to maximize their stability and PL QY may be expected to produce materials with broad applicability in FDT.

In this work, we first developed amorphous borondoped CNQDs (B-CNQDs) measuring approximately $5.0 \mathrm{~nm}$ in size via a hydrothermal process followed by the top-down cutting approach. Unlike conventional QDs and crystalline CNQDs, amorphous B-CNQDs demonstrate strong bright-blue PL emission intensity of millions scale under ultraviolet (UV) radiation, a high QY of $87.4 \%$, distinct excitation-independent emission behaviors, and unprecedented fluorescence stability (i.e., no obvious fading after two years). When the B-CNQDs are used in ink, the information of specific graphics rubbed onto commercially available paper could clearly be read under UV excitation but is invisible in ambient light. The amorphous B-CNQDs also display outstanding selectivity and sensitivity in the detection of $\mathrm{Fe}^{2+}$ and $\mathrm{Cd}^{2+}$ in aqueous solution at concentrations as low as $10^{-9} \mathrm{~mol} \mathrm{~L}^{-1}$. This work provides a new route for the rational design of high-performance FDT materials by using water-soluble amorphous carbon-based QDs.

\section{EXPERIMENTAL SECTION}

\section{Materials}

All of the reagents used in this work were of analytical grade and applied as received without further purification.

\section{Preparation of amorphous B-CNQDs}

Amorphous B-CNQDs were prepared by mixing citric acid, boric acid, and urea (molar ratio: 1:6:6) with $10 \mathrm{~mL}$ of deionized water under uniform grinding for $5 \mathrm{~min}$. Next, the mixture was placed in a $50-\mathrm{mL}$ Teflon-lined autoclave and heated at $180^{\circ} \mathrm{C}$ for $2 \mathrm{~h}$. After cooling to room temperature $\left(25^{\circ} \mathrm{C}\right)$ in air, the product was dissolved in water, sonicated for approximately $6 \mathrm{~h}$, passed through a $0.22-\mu \mathrm{m}$ filter membrane to discard large particles with a flake-like structure, and then re-purified. Finally, the filtrate was collected and diluted to prepare BCNQD suspensions $\left(0.5 \mathrm{mg} \mathrm{mL}^{-1}\right)$. The contrast samples were synthesized by maintaining all of the above conditions while changing the molar ratio of boric acid.

\section{Preparation of CNQDs}

The CNQDs were prepared by following the procedure applied to the preparation of the B-CNQDs but without boric acid; all other parameters were held constant. The obtained samples were denoted CNQDs.

\section{Preparation of bulk boron-doped graphitic carbon nitride} Bulk boron-doped graphitic carbon nitride $(\mathrm{B}-\mathrm{CN})$ was prepared by following the procedure applied to the preparation of the B-CNQDs but without ultrasound and filtration; all other parameters were held constant. The obtained samples were denoted B-CN.

\section{Characterization}

X-ray diffraction (XRD, Siemens D-5000) with $\mathrm{Cu} \mathrm{Ka}$ radiation $(\lambda=0.154 \mathrm{~nm})$ was used to assess the structural and textural properties of the B-CNQDs. Fourier transform infrared (FTIR) spectra in the wavelength range of $400-4000 \mathrm{~cm}^{-1}$ were recorded on an IRAffinity-1 FTIR spectrometer by using conventional $\mathrm{KBr}$ pellets. The chemical states of $\mathrm{C}, \mathrm{N}, \mathrm{O}$, and doped $\mathrm{B}$ elements were tested by X-ray photoelectron spectroscopy (XPS, PHI Quantera X-ray photoelectron spectrometer with $\mathrm{Al} \mathrm{Ka}$ radiation, $300 \mathrm{~W}$ ). Scanning electron microscopy (SEM) images of the precursor before filtration were obtained using a Hitachi S-4800 microscope. Transmission electron microscopy (TEM, FEI Tecai F20) was used to observe the nanostructures of the B-CNQDs. Atomic force microscopy (AFM) was performed on a Park Systems Corp. scanning probe microscopy system (Lui-Dong 90610, Suwon, South Korea). Room-temperature ultravioletvisible (UV-vis) absorption spectra were recorded on a UV-2450 Shimadzu system. The PL spectra were recorded on an F-2500 fluorescence spectrometer at room temperature. Time-resolved fluorescence (TRF) decay spectra were collected using a streak camera (C10910, Hamamatsu); here, the emission signal was reflected onto 
the streak camera by a mirror.

\section{Determination of fluorescence QYs}

The fluorescence QY was determined by a typical slope method with quinine sulfate as the reference [26]: comparing the integrated photoluminescence intensity (360 nm excitation) and the absorbance value (its maximal absorption intensity was controlled in the range of $0-0.1$ ) of the amorphous B-CNQDs with that of the references. Equation (1) was then applied:

$\Phi_{\mathrm{B}}=\Phi_{\mathrm{qs}}\left(K_{\mathrm{B}} / K_{\mathrm{qs}}\right)\left(\eta_{\mathrm{B}} / \eta_{\mathrm{qs}}\right)^{2}$,

where $\Phi$ is the $\mathrm{QY}, K$ is the slope determined by the curves, and $\eta$ is the refractive index. The subscripts "qs" and "B" refer to quinine sulfate and the B-CNQDs, respectively. For these aqueous solutions, $\eta_{\mathrm{B}(1.33)} / \eta_{\mathrm{qs}(1.33)}=$ 1. Thus, Equation (1) could be simplified to Equation (2): $\Phi_{\mathrm{B}}=\Phi_{\mathrm{qs}}\left(K_{\mathrm{B}} / K_{\mathrm{qs}}\right)$.

\section{Fluorescence selectivity and interference measurements}

Exactly $1 \mathrm{~mL}$ of the B-CNQD suspension $\left(0.5 \mathrm{mg} \mathrm{mL}^{-1}\right)$ was mixed with deionized water. Chlorate solutions of $\mathrm{Na}^{+}, \mathrm{K}^{+}, \mathrm{Ca}^{2+}, \mathrm{Cu}^{2+}, \mathrm{Fe}^{2+}, \mathrm{Ni}^{2+}, \mathrm{Sn}^{2+}, \mathrm{La}^{2+}, \mathrm{Ce}^{4+}$, and $\mathrm{Cd}^{2+}$ were then individually added to the suspension at a concentration of $20.0 \mu \mathrm{mol} \mathrm{L} \mathrm{L}^{-1}$ (final volume, $4 \mathrm{~mL}$ ). After reacting for $30 \mathrm{~min}$ under static conditions, fluorescence emission spectra were recorded at room temperature under an excitation wavelength of $360 \mathrm{~nm}$. The fluorescence intensity of B-CNQD solutions of different
$\mathrm{pH}$ (i.e., 1-14) was also recorded at room temperature under an excitation wavelength of $360 \mathrm{~nm}$.

\section{Fluorescence sensitivity detection of $\mathrm{Fe}^{2+}$ and $\mathrm{Cd}^{2+}$}

Fluorescence sensitivity was evaluated using the same procedures adopted for selectivity assessment, but the $\mathrm{Fe}^{2+}$ and $\mathrm{Cd}^{2+}$ concentrations were varied from 0 to $20.0 \mu \mathrm{mol} \mathrm{L}^{-1}$.

\section{RESULTS AND DISCUSSION}

The amorphous B-CNQDs were prepared by the hydrothermal method followed by the top-down cutting approach via ultrasound and filtration, the details of which are described in the EXPERIMENTAL SECTION. As shown in Scheme 1, after the hydrothermal reaction, B atoms are successfully doped into the carbon nitride $(\mathrm{CN})$ framework. The SEM image in Fig. S1a confirms that the as-synthesized B-CN has a bulk structure with a diameter of $\sim 5 \mu \mathrm{m}$, which agrees well with literature reports on this material $[35,36]$. The obtained bulk B-CN was then subjected to ultrasound treatment for $6 \mathrm{~h}$. During ultrasonication, the ordered in-plane packing and interfacial stacking of the $\mathrm{CN}$ structures were destroyed or decreased, thereby cutting the bulk material first into nanosheets and then into amorphous nanodots (Fig. S1a, d). As shown in the SEM images in Fig. S1c, d, the B-CNQDs mainly range from 20 to $30 \mathrm{~nm}$ in size, which is insufficient to achieve high quantum confinement effects.

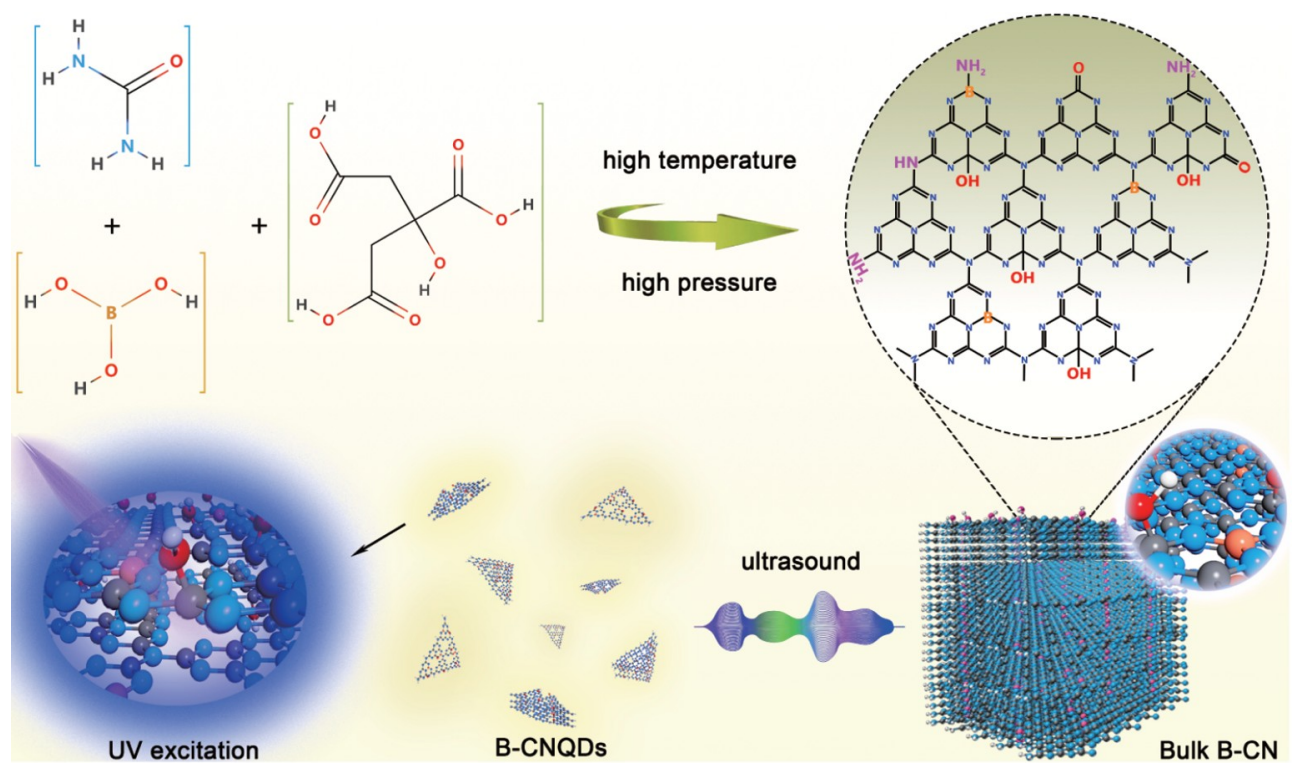

Scheme 1 Schematic of the synthesis process of the amorphous B-CNQDs. Urea, boric acid, and citric acid were used as precursor materials to prepare amorphous B-CNQDs by hydrothermal polymerization, ultrasound, and filtration. Amorphous B-CNQDs in an aqueous medium are faint yellow in color under ambient light and give a blue glow under a $360 \mathrm{~nm}$ ultraviolet lamp. 
Therefore, large-sized B-CNQDs should be removed from the aqueous solution, and the nanoparticles should be purified to obtain particle sizes of below $10 \mathrm{~nm}$. The TEM image in Fig. 1a shows uniformly distributed nanodots measuring only a few nanometers in size after filtration. The high-resolution TEM image in the inset in Fig. 1a reveals that the obtained B-CNQDs are amorphous in structure without detectable lattices. The particle size indicated by the Gaussian distribution histogram in Fig. $1 \mathrm{~b}$ reveals that the B-CNQDs have diameters ranging from 1 to $9 \mathrm{~nm}$, with an average size of $5 \mathrm{~nm}$. The AFM confirms the good dispersion of the B-CNQDs (Fig. 1c), which is well consistent with the TEM observations. Interestingly, the thickness of the nanodots varies from 0.612 to $3.307 \mathrm{~nm}$ (Fig. 1d and Fig. S2), thereby indicating that the amorphous B-CNQDs comprise 3-10 CN layers (the CNQD interlayer spacing, $0.32 \mathrm{~nm}$ ).

The XRD patterns of the bulk B-CN and the amorphous B-CNQDs reveal their structural and textural properties. As shown in Fig. 1e, two characteristic peaks at $27.4^{\circ}$ and $13.1^{\circ}$, which respectively correspond to the typical graphitic interlayer stacking of the (002) plane and the periodic in-plane structural packing feature of the (100) lattice plane of $\mathrm{CN}[37,38]$, could clearly be observed in the XRD pattern of bulk B-CN. In the XRD pattern of the B-CNQDs, the peak corresponding to (100) phase disappears completely and only a weak and broad peak at $27.1^{\circ}$ is observed, thus suggesting the destruction of the long-range in-plane ordering, decreased interplanar stacking, and increased stacking distance of the remaining layers $[4,39]$. The weak hump-like diffraction peak further confirms the amorphous nature of the BCNQDs, which is consistent with the TEM results.

The FTIR spectra were used to verify the surface groups and chemical structures of the amorphous B-CNQDs. As shown in Fig. 1f, the spectrum of the amorphous BCNQDs shows a peak at $790 \mathrm{~cm}^{-1}$, which could be associated with the characteristic breathing mode of tri-striazine rings and reveals the similarity of their structure to that of $\mathrm{CN}$ [3]. More importantly, compared with that of bulk B-CN $\left(810 \mathrm{~cm}^{-1}\right)$, the weak and left-shift peak corresponding to the tri-s-triazine rings implies the disruption of the in-plane structural packing motif of the BCNQDs, consistent with the XRD results. The peaks at 1398 and $1660 \mathrm{~cm}^{-1}$ are respectively attributed to the C$\mathrm{N}$ and $\mathrm{C}=\mathrm{N}$ bonds of the $\mathrm{C}-\mathrm{N}$ heterocycle $[40,41]$. The bands at 1112 and $1624 \mathrm{~cm}^{-1}$ are ascribed to the stretching vibrations of $\mathrm{C}-\mathrm{O}$ and $\mathrm{C}=\mathrm{O}$ [27], while the broad and intensified peak in the range of $3000-3500 \mathrm{~cm}^{-1}$ is assigned to the stretching vibrations of
$\mathrm{N}-\mathrm{H}$ or $\mathrm{O}-\mathrm{H}$ in uncondensed amino and hydroxyl groups [42]. This result suggests that the surface of the obtained amorphous B-CNQDs is rich in multiple hydrophilic groups. The weak band at $1260 \mathrm{~cm}^{-1}$ is attributed to the stretching mode of $\mathrm{C}-\mathrm{N}-\mathrm{B}$, thereby indicating the successful incorporation of $\mathrm{B}$ atoms into the CNQD framework [43-46].

The chemical composition of the amorphous BCNQDs was analyzed by XPS. The XPS full-survey scan spectrum of the dots is shown in Fig. 2a and reveals the coexistence of $\mathrm{B}, \mathrm{C}, \mathrm{N}$, and $\mathrm{O}$ elements in the dots. The weak $\mathrm{O} 1$ s peak located at approximately $532 \mathrm{eV}$ may be ascribed to the surface hydroxylation of the B-CNQDs $[35,42,47]$. The $\mathrm{B}, \mathrm{C}, \mathrm{N}$, and $\mathrm{O}$ atomic percentages obtained from XPS are $1.24 \%, 39.76 \%, 54.47 \%$ and $4.53 \%$, respectively, and the $\mathrm{C} / \mathrm{N}$ ratio is 0.73 (Table $\mathrm{S} 1$ ), which is consistent with the typical theoretical value for $\mathrm{CN}$. The high-resolution $B$ 1s spectrum in Fig. $2 \mathrm{~b}$ demonstrates the presence of C-N-B (191.6 eV; B is surrounded by three $\mathrm{N}$ atoms) [48,49], which is in accordance with the FTIR observations and indicates the successful substitution of $\mathrm{B}$ atoms into the carbon sites of the CNQD framework.

The high-resolution XPS spectra of C 1s, N 1s, and O 1s were analyzed to investigate the effect of $\mathrm{B}$ doping on the structure of the $\mathrm{CN}$ framework. As illustrated in Fig. 2c, the two main peaks observed at 284.6 and $288.0 \mathrm{eV}$ could be ascribed to the pure graphitic species of $\mathrm{C}-\mathrm{C} / \mathrm{C}=\mathrm{C}$ bonds in carbon impurities and $\mathrm{sp}^{2}$-hybridized carbon atoms bonded to the aliphatic amine in the aromatic rings $(\mathrm{N}-\mathrm{C}=\mathrm{N})$ [50-52], respectively. The weak peaks located at 286.1 and $289.1 \mathrm{eV}$ are respectively attributed to the C$\mathrm{NH}_{x}(x=1,2)$ groups on the edges of heptazine units and $\mathrm{C}-\mathrm{O}$ bonds at the edges of the B-CNQDs $[27,40]$. The peak ratios of $\mathrm{C}-\mathrm{NH}_{x}$ and $\mathrm{C}-\mathrm{O}$ calculated through $\mathrm{C} 1 \mathrm{~s}$ are $7.06 \%$ and $2.43 \%$ (Table S2), respectively, thus reaffirming the enrichment of amino and hydroxyl groups in the amorphous B-CNQDs. The N 1s XPS spectrum of the B-CNQDs could be divided into four major peaks located at approximately $398.4,399.6,400.8$, and $403.9 \mathrm{eV}$ (Table S3). As shown in Fig. 2d, the intense peak located at $398.4 \mathrm{eV}$ corresponds to the $\mathrm{sp}^{2}$-hybridized aromatic groups in tri-s-triazine rings $\left(\mathrm{N}-(\mathrm{C})_{2}\right)$, while the weak peak at $399.6 \mathrm{eV}$ is attributed to the tertiary bridging $\mathrm{N}$ atoms in $\mathrm{N}-(\mathrm{C})_{3}$ (where the $\mathrm{N}$ atom is surrounded by three $\mathrm{C}$ atoms) groups [28]. The peak with a binding energy of $400.8 \mathrm{eV}$ suggests the presence of amino groups carrying hydrogen $(\mathrm{N}-\mathrm{H})$, thus implying the enrichment of amino groups in the B-CNQDs. The weak peak centered at $403.9 \mathrm{eV}$ corresponds to charging effects or positive charge localization in heterocycles [53,54]. 

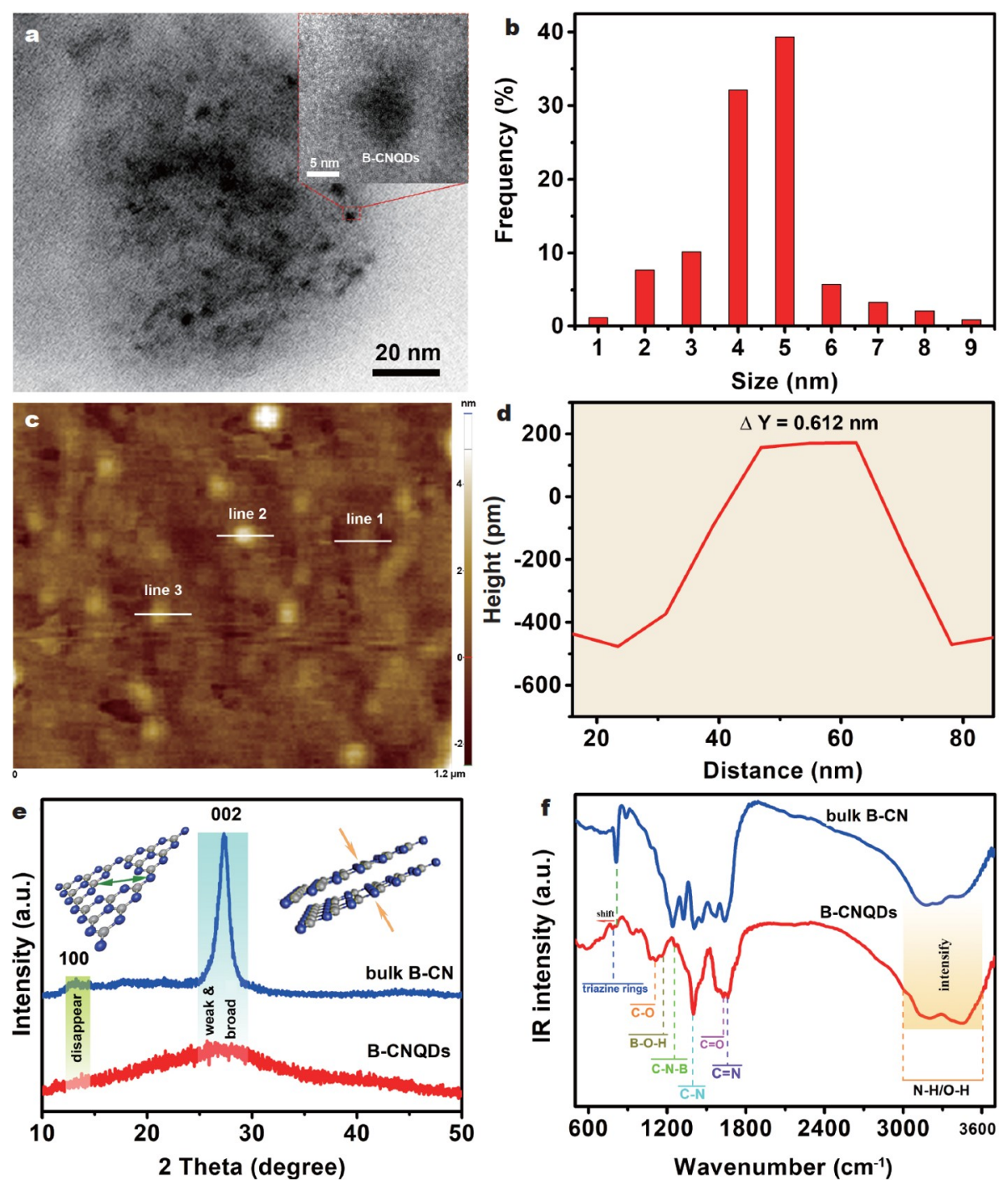

Figure 1 Structural characterization of the amorphous B-CNQDs. (a) TEM image of the amorphous B-CNQDs. Inset: corresponding highresolution TEM image. (b) Particle size distribution obtained from the TEM results. (c) AFM image and (d) the corresponding height image of the amorphous B-CNQDs. (e) XRD patterns and (f) FTIR spectrum of the amorphous B-CNQDs.

Deconvolution of the O 1s core-level spectrum (Fig. 2e) suggests the presence of two chemically distinct species: $\mathrm{O}-\mathrm{H}(533.0 \mathrm{eV})$ resulting from the surface dissociation of water, and $\mathrm{C}-\mathrm{O}(531.5 \mathrm{eV})$, which originates from the $\mathrm{C}$ atom-bonded surface hydroxyl groups $(\mathrm{C}-\mathrm{O}-\mathrm{H})[42,47]$. These findings agree well with the FTIR results and affirm the presence of surface hydroxyl groups on the B-CNQDs (Table S4). Taken together, the TEM, XRD, FTIR, and XPS characterizations confirm that the as-prepared BCNQDs consist of the main framework of amorphous CNQDs and doped B atoms with surface enrichment of amino and hydroxyl groups (Fig. 2f).

The optical properties of the amorphous B-CNQDs in their aqueous solutions were detected by UV-vis diffuse reflectance and PL spectroscopy. Fig. 3a shows a strong absorption edge and characteristic peak located at 250 and $335 \mathrm{~nm}$, respectively. The first edge, which corresponds to the $\pi-\pi^{*}$ electronic transitions of aromatic $\mathrm{sp}^{2}$ domains, produces nearly no fluorescence signal, and the second peak could be attributed to the $n-\pi^{*}$ electronic transitions of $\mathrm{C}-\mathrm{O}-\mathrm{H}, \mathrm{C}=\mathrm{N}$, and $\mathrm{C}-\mathrm{N}-\mathrm{B}$ in the $\mathrm{B}-$ CNQDs $[25,27,55]$. Upon UV light excitation, a strong blue fluorescence emission peak centered at $440 \mathrm{~nm}$ is observed in B-CNQDs because of the trapping of excitedstate energy by the surface states [20].

The B-CNQDs demonstrate a Stokes shift of approxi- 

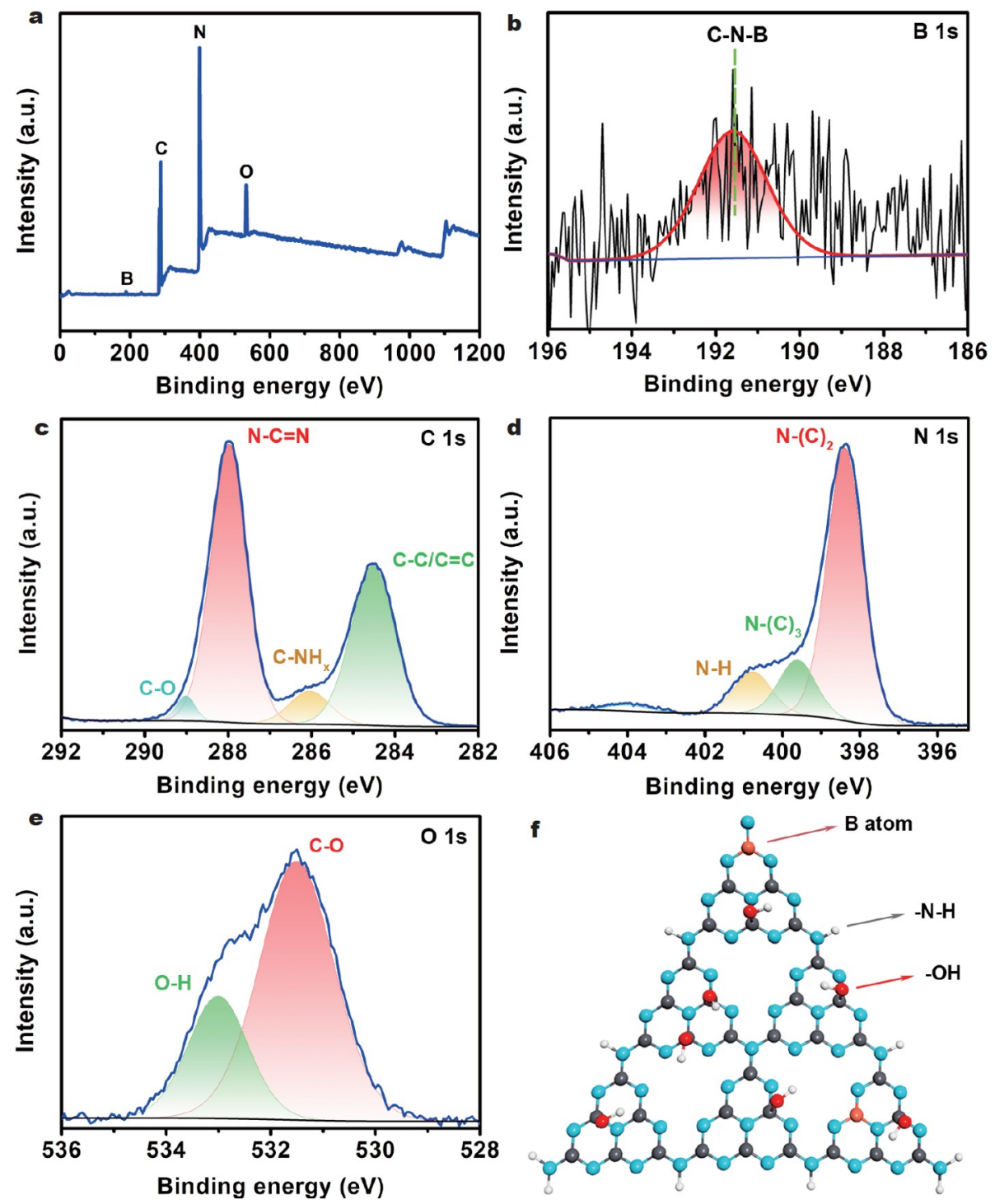

Figure 2 Surface chemical compositions and states of B, C, N and O elements in the amorphous B-CNQDs. (a) XPS survey scan of the amorphous B-CNQDs. (b-e) High-resolution XPS spectra of B 1s, C 1s, N 1s, and O 1s of the amorphous B-CNQDs. Each spectrum was deconvoluted by fitting with Gaussian functions. (f) Proposed structure of the amorphous B-CNQDs (B, C, N, O and H atoms are respectively indicated as orange, gray, blue, red, and white spheres in the atomic model).

mately $105 \mathrm{~nm}$, which could effectively improve the fluorescence efficiency of the dots owing to the drop in reabsorption events leading to nonradiative recombination [26]. The anti-Stokes excitation peak of the dots appears at $343 \mathrm{~nm}$ and could be ascribed to up-conversion emission mechanisms. The as-synthesized B-CNQDs emit a faint yellow glow under visible light and brightblue luminescence under UV $(360 \mathrm{~nm})$ excitation (inset, Fig. 3a and Fig. S3). Interestingly, the optimized BCNQDs show extremely strong PL signals (citric acid: boric acid:urea ratio, 1:6:6), accompanied by excitation- independent wavelength behaviors different from those of most other reported CNQDs. As shown in Fig. 3b, the assynthesized B-CNQDs exhibit a strong emission peak centered at approximately $440 \mathrm{~nm}$, and no peak shift is observed in the PL spectra obtained upon excitation at $\lambda$ $=300-420 \mathrm{~nm}$. The strongest emission is achieved under $360 \mathrm{~nm}$ excitation. Pure CNQDs show fluorescence emission spectra and excitation-dependent behavior at different excitation wavelengths (Fig. S4), along with a sharp decrease in PL intensity. The excitation-dependent wavelength behavior of QDs is generally caused by the 

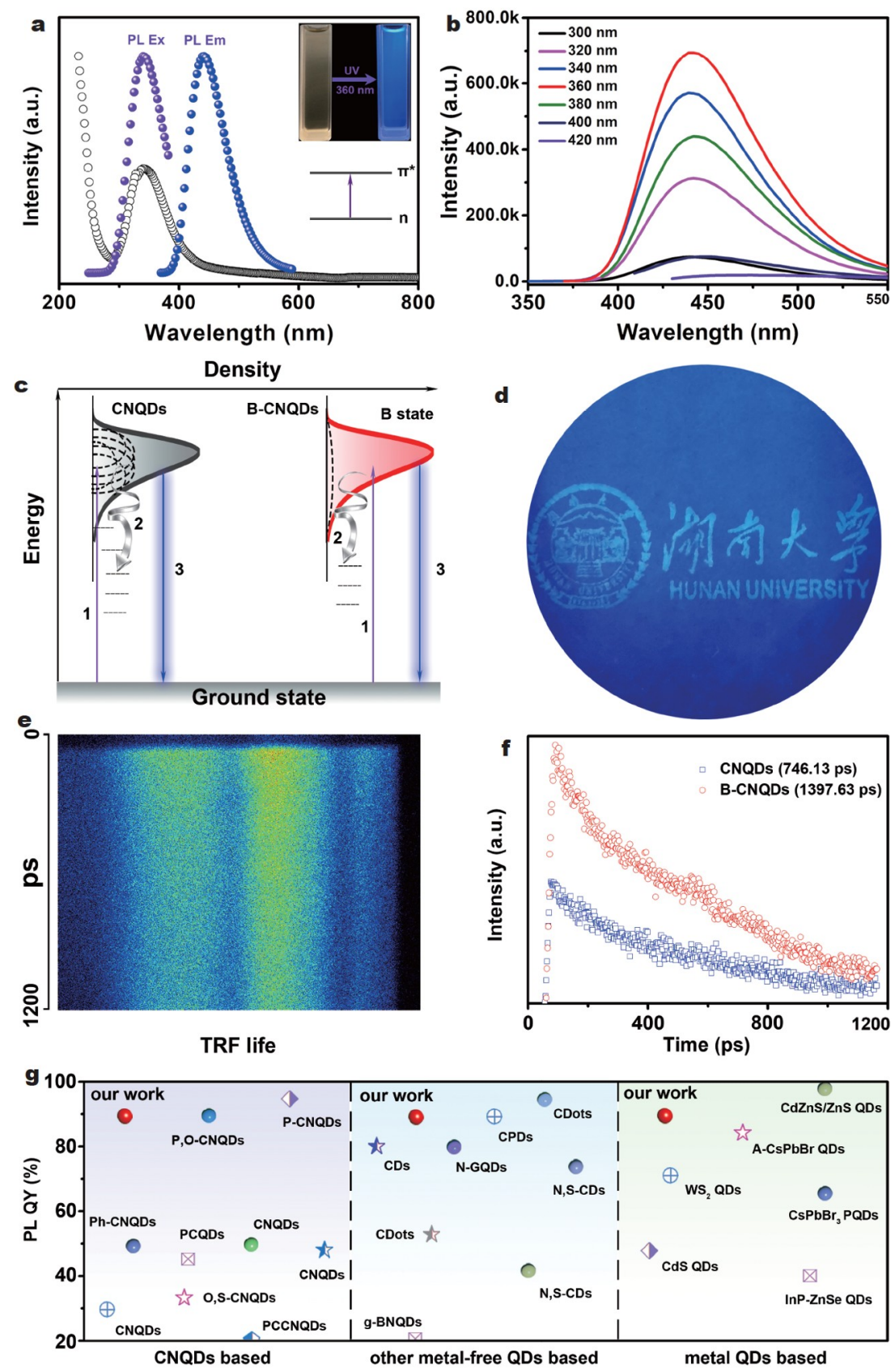

Figure 3 Optical characterization of the amorphous B-CNQDs. (a) Normalized UV-vis absorption (black) and PL excitation (violet) and emission (blue) fluorescence spectra of the amorphous B-CNQDs. (b) The PL fluorescence spectra of the amorphous B-CNQDs at different excitation wavelengths ranging from 300 to $420 \mathrm{~nm}$. (c) Representation of the fluorescence mechanism of the non-doped CNQDs and amorphous B-CNQDs: 1) electrons are excited from the ground-state and trapped by the surface states, 2) excited electrons return to the ground-state via a nonradiative route, and 3) excited electrons return to the ground-state via a radiative route. (d) Fluorescence images obtained under ultraviolet excitation (360 $\mathrm{nm}$ ) when the amorphous B-CNQDs were used as ink. (e) TRF lifetime of the B-CNQDs and (f) TRF spectra of the pristine CNQDs and the amorphous BCNQDs. The spectra are recorded under the excitation (wavelength, $400 \mathrm{~nm}$ ) of a picosecond pulsed light-emitting diode at room temperature. (g) Comparison of the fluorescence QYs of the amorphous B-CNQDs and other reported QDs (specific parameters are given in Table S5).

size effect resulting from quantum dimensions and the inhomogeneous distribution of the surface states, re- spectively $[9,20]$. In our work, however, the TEM and size statistics images in Fig. 1a, b indicate that the size of the 
B-CNQDs is not uniform. Hence, the excitation-independent behavior of B-CNQDs may be ascribed to the uniform surface states introduced by B doping (labeled as the B-state; Fig. 3c); in this case, electrons trapped by the newly formed surface states are able to facilitate a high level of radiative recombination.

Interestingly, the bright-blue fluorescence of specific graphics on commercially available paper (the paper showed no background UV fluorescence) could easily be observed under UV excitation ( $360 \mathrm{~nm}$ ) but is invisible in ambient light when the amorphous B-CNQDs were used as ink (Fig. 3d and Fig. S5). This finding demonstrates the promising applications of the dots in imaging ink and biolabeling. Moreover, the observed blue fluorescence shows no significant fading after two years (Fig. S6), thereby indicating the hyperstability of the B-CNQDs, which could be attributed to their amorphous structure. The effective separation and lifetime of photoexcited electrons over the amorphous B-CNQDs could be confirmed by TRF decay spectroscopy (Fig. 3e, f and Fig. S7). The average TRF lifetime $(\tau)$ calculated by the equation: $\tau=\left(A_{1} \tau_{1}{ }^{2}+A_{2} \tau_{2}{ }^{2}\right) /\left(A_{1} \tau_{1}+A_{2} \tau_{2}\right)$ is 1397.63 ps for the amorphous B-CNQDs and 746.13 ps for the pristine CNQDs. The fluorescence QY of the B-CNQDs excited by $360 \mathrm{~nm}$ UV light was calculated to be $87.4 \%$ when quinine sulfate was used as a standard (54\%); this QY is much higher than that of pure CNQDs (49.5\%) and exceeds those of most other QDs reported thus far (Fig. $3 \mathrm{~g}$ and Table S5). These results confirm that B doping into the amorphous structures of the CNQDs can synergistically optimize the optical properties of the resultant materials and prevent fluorescence quenching to achieve high fluorescence QY.

The complex fluorescence mechanism of QDs renders identification of the relevant microprocess difficult but two hypotheses, namely, electronic conjugate structures and emissive traps [20,56,57], have been proposed. In general, the $\pi-\pi^{*}$ electronic transitions of aromatic $\mathrm{sp}^{2}$ domains in the $\mathrm{B}-\mathrm{CNQDs}$ are unable to produce a fluorescent signal (Fig. 3a), thereby indicating the fluorescence mechanism of the B-CNQDs is not directly related to their electronic structures. In the hypothesis of emissive traps, the uniform surface states brought about by the appropriate amount of $\mathrm{B}$ doping should have a prominent impact on the fluorescence emission of the dots, resulting in the radiative recombination of electrons and holes trapped on the B-CNQD surface [20,58]. We tested the fluorescence spectra of B-CNQDs with different $\mathrm{B}$ doping contents in the excitation wavelength range from 300 to $420 \mathrm{~nm}$ to confirm the feasibility of this explanation. As shown in Fig. S8, distinct excitation-dependent fluorescence behaviors are observed when the $B$ contents in the CNQDs are regulated. The fluorescence intensity sharply decreases to tens of thousands units compared with the amorphous B-CNQDs with the appropriate amount of doped $\mathrm{B}$ atoms (Fig. 3b). The absorption spectra of these QDs depicted in Fig. S8d prove that the fluorescence mechanism depends on surface states, rather than electronic conjugate structures. The surface states of the B-CNQDs should be rather inconsistent because of their excitation-dependent behaviors. Thus, we propose that the strong and independent-excitation wavelength fluorescence emission of the BCNQDs is mainly attributed to the appropriate surfacedoping of $\mathrm{B}$ atoms.

The QDs, such as those obtained from carbon and graphene, generally show $\mathrm{pH}$-sensitive fluorescence behaviors $[4,18,59]$. As shown in Fig. 4a, the normalized fluorescence intensity of the amorphous B-CNQDs is highly sensitive to the solution $\mathrm{pH}$ and increases sharply with an increase in $\mathrm{pH}$ from 2 to 5 , remains stable at $\mathrm{pH}$ 5-11, and then decreases at $\mathrm{pH} 12-14$. The effect of $\mathrm{pH}$ can be understood in terms of the change in surface charge owing to protonation-deprotonation. In this scenario, the dependence of fluorescence on the $\mathrm{pH}$ values suggests that fluorescent species in the amorphous BCNQDs probably have alkalescence sites since the fluorescence is quenched in acidic and strong alkali media, consistent with earlier findings (FTIR, XPS) that the BCNQDs contain large numbers of amino and hydroxyl groups.

The prospective use of the obtained amorphous BCNQDs as sensors for metal ion detection was assessed. As shown in Fig. 4b, the fluorescence signals of the BCNQDs were detected before and after the addition of perchlorate salts containing various metal ions (such as $\mathrm{Na}^{+}, \mathrm{K}^{+}, \mathrm{Ca}^{2+}, \mathrm{Cu}^{2+}, \mathrm{Fe}^{2+}, \mathrm{Ni}^{2+}, \mathrm{Sn}^{2+}, \mathrm{La}^{2+}, \mathrm{Ce}^{4+}$, and $\mathrm{Cd}^{2+}$ ) at a single concentration of $20 \mu \mathrm{mol} \mathrm{L}{ }^{-1}$. The sensitivity of the fluorescence intensity of the B-CNQDs strongly relies on the metal ions in the same solution. The undetectable fluorescence quenching of B-CNQDs in the presence of $\mathrm{Na}^{+}, \mathrm{K}^{+}, \mathrm{Ni}^{2+}$, and $\mathrm{Ce}^{4+}$ and the weak fluorescence quenching observed after the addition of $\mathrm{Ca}^{2+}, \mathrm{Cu}^{2+}, \mathrm{Sn}^{2+}$, and $\mathrm{La}^{2+}$ could be attributed to nonspecific interactions between the amino and hydroxyl groups of the dots and the metal ions. In particular, the fluorescence intensity of the B-CNQDs is remarkably quenched in the presence of $\mathrm{Fe}^{2+}$ and $\mathrm{Cd}^{2+}$. Fig. 4c shows a photograph of the $\mathrm{B}-$ CNQD solution after the addition of different metal ions under ambient light and UV irradiation $(360 \mathrm{~nm})$. The B- 

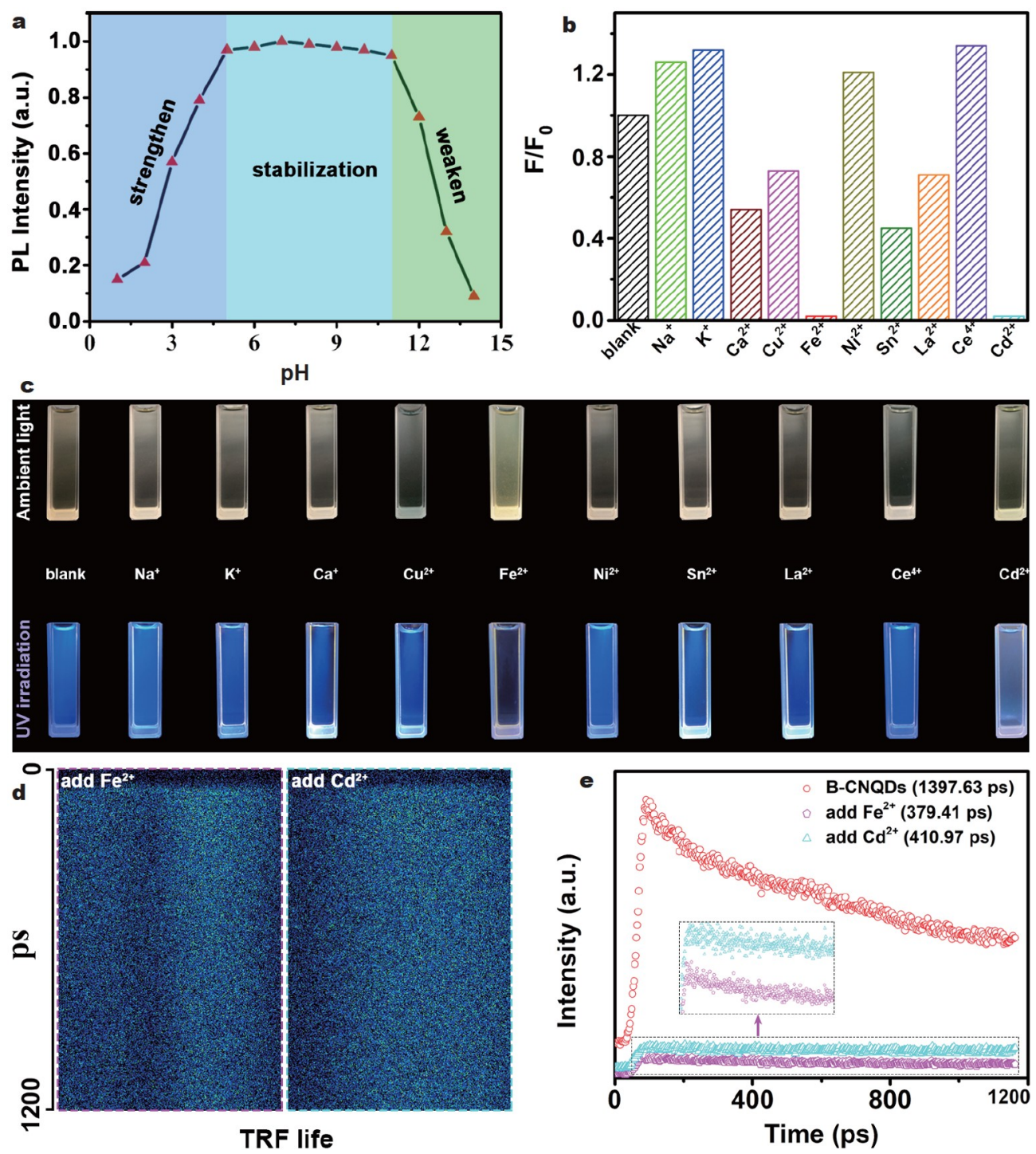

Figure 4 Selectivity characterization of the amorphous B-CNQDs. (a) pH-sensitive fluorescence behaviors of the amorphous B-CNQDs. (b) Fluorescence responses of the amorphous B-CNQDs in the presence of different metal ions $\left(\lambda_{\mathrm{ex}}=360 \mathrm{~nm},\left[\mathrm{M}^{n+}\right]=20 \mu \mathrm{mol} \mathrm{L}{ }^{-1}\right)$. (c) Photographs of the B-CNQD solutions after the addition of different metal ions under ambient light (top row) and $360 \mathrm{~nm}$ ultraviolet irradiation (bottom row). (d, e) TRF lifetimes and the comparison of the TRF spectra of amorphous B-CNQDs in the presence of $\mathrm{Fe}^{2+}$ and $\mathrm{Cd}^{2+}$. The spectra are recorded under the excitation (wavelength, $400 \mathrm{~nm}$ ) of a picosecond pulsed light-emitting diode at room temperature.

CNQD solutions exhibit different colors because of the intrinsic properties of the different metal ions, and the fluorescence intensity of these samples varies greatly under UV irradiation. The fluorescence of the solutions was nearly completely quenched after the addition of $\mathrm{Fe}^{2+}$ and $\mathrm{Cd}^{2+}$, consistent with the fluorescence intensity histogram (Fig. $4 \mathrm{~b}$ ) measured by fluorescence spectrometry. The TRF lifetimes in Fig. 4d, e also strongly demonstrate fluorescence quenching; specifically, the lifetimes of the B-CNQDs are sharply attenuated from $1397.63 \mathrm{ps}$ (Fig. 3e) to 379.41 and $410.97 \mathrm{ps}$ in the presence of $\mathrm{Fe}^{2+}$ and $\mathrm{Cd}^{2+}$, respectively. $\mathrm{Fe}^{2+}$, an important component of hemoglobin, is essential for oxygen delivery in the human body. An imbalance between the supply and demand for $\mathrm{Fe}^{2+}$ could result in various diseases, such as anemia, cardiovascular disease and Alzheimer's disease. The wide use of $\mathrm{Cd}^{2+}$ in the mining, smelting, dye, battery and chemical industries may present great harm to the human body on account of the strong toxicity and bioaccumulation of the metal even at very low concentrations [6062]. Thus, the amorphous B-CNQDs show great potential use for the selective detection of $\mathrm{Fe}^{2+}$ and $\mathrm{Cd}^{2+}$. 

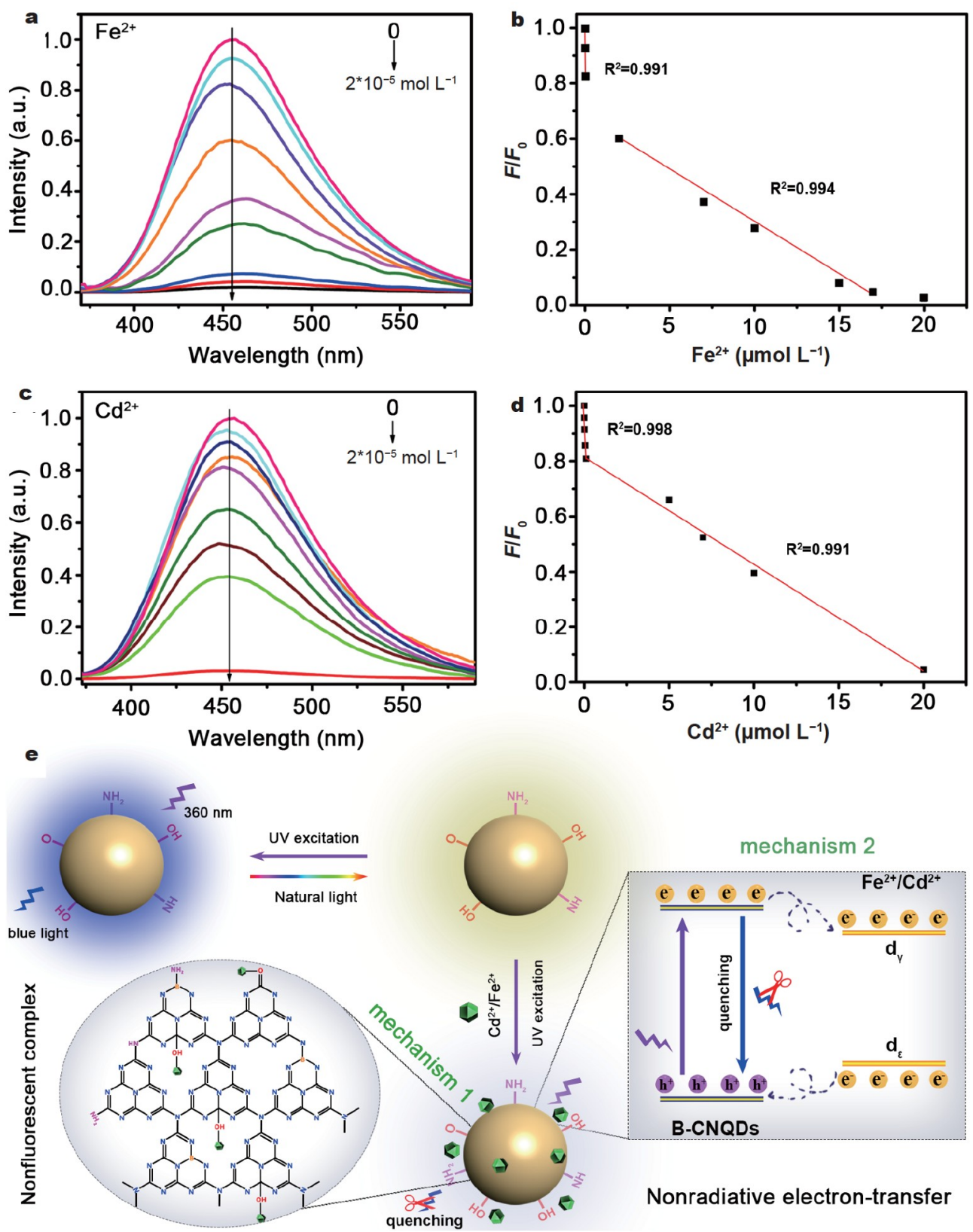

Figure 5 Sensitivity analysis of the amorphous B-CNQDs. Change in fluorescence intensity of the amorphous B-CNQDs in water upon the addition of (a) $\mathrm{Fe}^{2+}$ and (c) $\mathrm{Cd}^{2+}$ (excitation wavelength, $360 \mathrm{~nm}$ ). Stern-Volmer plots for the fluorescence quenching of the amorphous B-CNQDs with (b) $\mathrm{Fe}^{2+}$ and (d) $\mathrm{Cd}^{2+}$ in water, showing the variance of $F_{0} / F$ with $\mathrm{Fe}^{2+}$ and $\mathrm{Cd}^{2+}$. (e) Proposed fluorescence quenching mechanisms of the B-CNQDs in the presence of $\mathrm{Fe}^{2+}$ and $\mathrm{Cd}^{2+}$ ions.

We tested the fluorescence responses of the amorphous $\mathrm{B}-\mathrm{CNQD}$ in the absence and presence of $\mathrm{Fe}^{2+}$ and $\mathrm{Cd}^{2+}$ at the concentrations of $0-20 \mu \mathrm{mol} \mathrm{L} \mathrm{L}^{-1}$ to highlight the detection sensitivity of the sensor for the target ions. As shown in Fig. 5a, c, the fluorescence signals of the BCNQDs significantly decrease with an increase in $\mathrm{Fe}^{2+}$ and $\mathrm{Cd}^{2+}$ concentration, thus indicating the high sensi- tivity of the dots toward $\mathrm{Fe}^{2+}$ and $\mathrm{Cd}^{2+}$. Photographs of the color of the above solutions under ambient light and UV irradiation $(360 \mathrm{~nm})$ are shown in Figs S9 and S10. The fluorescence quenching of the B-CNQDs by $\mathrm{Fe}^{2+}$ and $\mathrm{Cd}^{2+}$ ions could be interpreted by Stern-Volmer analysis. The quenching efficiency of the dots may be well fitted by the following Stern-Volmer equation: $F_{0} / F=1+K_{\mathrm{sv}} Q$, 
where $F_{0}$ and $F$ are the PL intensities of the B-CNQDs in the absence and presence of $\mathrm{Fe}^{2+}$ and $\mathrm{Cd}^{2+}$, respectively, $Q$ is the concentration of $\mathrm{Fe}^{2+}$ or $\mathrm{Cd}^{2+}$, and $K_{\mathrm{sv}}$ is the SternVolmer constant. The linear dependence of the fluorescence quenching intensity on the concentration of $\mathrm{Fe}^{2+}$ or $\mathrm{Cd}^{2+}$ ions is clearly observed in the Stern-Volmer plots shown in Fig. 5b, d. The fluorescence of the pristine BCNQDs could be completely quenched by the addition of $20 \mu \mathrm{mol} \mathrm{L}^{-1} \mathrm{Fe}^{2+}$ and $20 \mu \mathrm{mol} \mathrm{L}{ }^{-1} \mathrm{Cd}^{2+}$. Moreover, the BCNQDs show wide linearity ranging from 0 to $20 \mu \mathrm{mol} \mathrm{L}^{-1}$ and good sensitivity with low detection limits of 2.3 and $1.1 \mathrm{nmol} \mathrm{L}^{-1}$ (calculated by the $3 \sigma$ rule [63]) for $\mathrm{Fe}^{2+}$ and $\mathrm{Cd}^{2+}$, respectively, at a signal-to-noise ratio of 3 . These values indicate that the sensor is sufficiently sensitive to meet the limits of $\mathrm{Fe}$ and $\mathrm{Cd}$ in drinking water established by the U.S. Environmental Protection Agency (5.537 and $1.8 \mu \mathrm{mol} \mathrm{L}{ }^{-1}$, respectively). The outstanding selectivity and fluorescence quenching of the synthesized dots may be ascribed to their formation of a stable nonfluorescent complex via the strong thermodynamic affinity and rapid chelation of $\mathrm{Fe}^{2+}$ and $\mathrm{Cd}^{2+}$ with the hydroxyl groups on the surface of the B-CNQDs (Fig. 5e, mechanism 1) and/or nonradiative electron-transfer involving the partial transfer of an electron in the excitedstate to the d orbital of $\mathrm{Fe}^{2+} / \mathrm{Cd}^{2+}$ (Fig. 5e, mechanism 2) $[2,4,64-66]$. The excellent performance of the B-CNQDs reflects their suitable application as a fluorescent sensor for the quantitative analysis of $\mathrm{Fe}^{2+}$ and $\mathrm{Cd}^{2+}$ in an aqueous solution.

\section{CONCLUSIONS}

In summary, we successfully fabricated novel amorphous B-CNQDs via the hydrothermal method followed by the top-down cutting approach. The synergistic effects of the uniform surface $B$ states and amorphous structures of the B-CNQDs significantly contributed to the latter's outstanding excitation-independent emission behavior and high blue PL QY of near $90 \%$, which is much higher than that of pure CNQDs (49.5\%) and most other QDs reported thus far. Systematic investigations indicate that the high structural disorder of the amorphous structures of the B-CNQDs not only endows them with superior elastic strain performance over a wide range of $\mathrm{pH}$, but also effectively reduces exciton quenching, thereby improving their fluorescent stability and PL QY. Interestingly, tailoring of the B doping content results in the B-CNQDs exhibiting excitation-independent emission behaviors via the formation of uniform B states, as well as ultra-strong fluorescent emission signals; these observations indicate that the amorphous $\mathrm{B}-\mathrm{CNQDs}$ are very promising fluorescent materials for FDT. For example, when used as ink, the amorphous B-CNQDs emitted a blue glow on commercial paper; the dots could also be used as a sensitive fluorescence sensor for detecting $\mathrm{Fe}^{2+}$ and $\mathrm{Cd}^{2+}$ in aqueous solutions with a detection limit of as low as $10^{-9} \mathrm{~mol} \mathrm{~L}^{-1}$. Our study provides a new route and scientific insights into the design of high-fluorescence-QY amorphous carbon-based materials, with potential applications in optical imaging, sensing and biolabeling.

Received 1 April 2021; accepted 8 May 2021;

published online 30 June 2021

1 Xu Y, Wang X, Zhang WL, et al. Recent progress in two-dimensional inorganic quantum dots. Chem Soc Rev, 2018, 47: 586-625

2 Liu S, Tian J, Wang L, et al. Hydrothermal treatment of grass: A low-cost, green route to nitrogen-doped, carbon-rich, photoluminescent polymer nanodots as an effective fluorescent sensing platform for label-free detection of $\mathrm{Cu}(\mathrm{II})$ ions. Adv Mater, 2012, 24: 2037-2041

3 Song Z, Lin T, Lin L, et al. Invisible security ink based on watersoluble graphitic carbon nitride quantum dots. Angew Chem Int Ed, 2016, 55: 2773-2777

4 Zhu S, Meng Q, Wang L, et al. Highly photoluminescent carbon dots for multicolor patterning, sensors, and bioimaging. Angew Chem Int Ed, 2013, 52: 3953-3957

5 Wang L, Xu D, Gao J, et al. Semiconducting quantum dots: Modification and applications in biomedical science. Sci China Mater, 2020, 63: 1631-1650

6 Gao W, Song H, Wang X, et al. Carbon dots with red emission for sensing of $\mathrm{Pt}^{2+}, \mathrm{Au}^{3+}$, and $\mathrm{Pd}^{2+}$ and their bioapplications in vitro and in vivo. ACS Appl Mater Interfaces, 2018, 10: 1147-1154

7 Gu B, Zhang Q. Recent advances on functionalized upconversion nanoparticles for detection of small molecules and ions in biosystems. Adv Sci, 2018, 5: 1700609

8 Duan J, Zhan J. Recent developments on nanomaterials-based optical sensors for $\mathrm{Hg}^{2+}$ detection. Sci China Mater, 2015, 58: 223240

9 Sun YP, Zhou B, Lin Y, et al. Quantum-sized carbon dots for bright and colorful photoluminescence. J Am Chem Soc, 2006, 128: 7756-7757

10 Zhu S, Song Y, Wang J, et al. Photoluminescence mechanism in graphene quantum dots: Quantum confinement effect and surface/ edge state. Nano Today, 2017, 13: 10-14

11 Strauss V, Margraf JT, Dolle C, et al. Carbon nanodots: Toward a comprehensive understanding of their photoluminescence. J Am Chem Soc, 2014, 136: 17308-17316

12 Jasieniak J, Califano M, Watkins SE. Size-dependent valence and conduction band-edge energies of semiconductor nanocrystals. ACS Nano, 2011, 5: 5888-5902

13 Ratnesh RK. Hot injection blended tunable CdS quantum dots for production of blue led and a selective detection of $\mathrm{Cu}^{2+}$ ions in aqueous medium. Optics Laser Tech, 2019, 116: 103-111

14 Zhang Y, Xiao JY, Zhu Y, et al. Fluorescence sensor based on biosynthetic CdSe/CdS quantum dots and liposome carrier signal amplification for mercury detection. Anal Chem, 2020, 92: 39903997

15 Hao L, Chen X, Liu D, et al. Charge transfer dynamics of the CdTe 
quantum dots fluorescence quenching induced by ferrous (II) ions. Appl Phys Lett, 2020, 116: 012105

16 Ran $\mathrm{X}$, Sun $\mathrm{H}, \mathrm{Pu}$ F, et al. Ag nanoparticle-decorated graphene quantum dots for label-free, rapid and sensitive detection of $\mathrm{Ag}^{+}$ and biothiols. Chem Commun, 2013, 49: 1079-1081

17 Yan Y, Gong J, Chen J, et al. Recent advances on graphene quantum dots: From chemistry and physics to applications. Adv Mater, 2019, 31: 1808283

18 Bian S, Shen C, Qian Y, et al. Facile synthesis of sulfur-doped graphene quantum dots as fluorescent sensing probes for $\mathrm{Ag}^{+}$ions detection. Sens Actuat B-Chem, 2017, 242: 231-237

19 Yang Z, Li Z, Xu M, et al. Controllable synthesis of fluorescent carbon dots and their detection application as nanoprobes. NanoMicro Lett, 2013, 5: 247-259

20 Dong Y, Pang H, Yang HB, et al. Carbon-based dots co-doped with nitrogen and sulfur for high quantum yield and excitation-independent emission. Angew Chem Int Ed, 2013, 52: 7800-7804

21 Dong $\mathrm{Y}$, Wang Q, Wu H, et al. Graphitic carbon nitride materials: Sensing, imaging and therapy. Small, 2016, 12: 5376-5393

22 Wang X, Sun G, Li N, et al. Quantum dots derived from twodimensional materials and their applications for catalysis and energy. Chem Soc Rev, 2016, 45: 2239-2262

23 Wang A, Wang C, Fu L, et al. Recent advances of graphitic carbon nitride-based structures and applications in catalyst, sensing, imaging, and leds. Nano-Micro Lett, 2017, 9: 47

24 Wang $\mathrm{T}$, Nie $\mathrm{C}$, Ao Z, et al. Recent progress in $\mathrm{g}-\mathrm{C}_{3} \mathrm{~N}_{4}$ quantum dots: Synthesis, properties and applications in photocatalytic degradation of organic pollutants. J Mater Chem A, 2020, 8: 485-502

$25 \mathrm{Wu}$ J, Yang S, Li J, et al. Electron injection of phosphorus doped g$\mathrm{C}_{3} \mathrm{~N}_{4}$ quantum dots: Controllable photoluminescence emission wavelength in the whole visible light range with high quantum yield. Adv Opt Mater, 2016, 4: 2095-2101

26 Cui Q, Xu J, Wang X, et al. Phenyl-modified carbon nitride quantum dots with distinct photoluminescence behavior. Angew Chem Int Ed, 2016, 55: 3672-3676

27 Zhou Z, Shen Y, Li Y, et al. Chemical cleavage of layered carbon nitride with enhanced photoluminescent performances and photoconduction. ACS Nano, 2015, 9: 12480-12487

28 Chen $\mathrm{X}, \mathrm{Liu} \mathrm{Q}, \mathrm{Wu} \mathrm{Q}$, et al. Incorporating graphitic carbon nitride $\left(\mathrm{g}-\mathrm{C}_{3} \mathrm{~N}_{4}\right)$ quantum dots into bulk-heterojunction polymer solar cells leads to efficiency enhancement. Adv Funct Mater, 2016, 26: 1719-1728

29 An T, Tang J, Zhang Y, et al. Photoelectrochemical conversion from graphitic $\mathrm{C}_{3} \mathrm{~N}_{4}$ quantum dot decorated semiconductor nanowires. ACS Appl Mater Interfaces, 2016, 8: 12772-12779

30 He X, Liu Y, Butch CJ, et al. One-pot exfoliation of graphitic $\mathrm{C}_{3} \mathrm{~N}_{4}$ quantum dots for blue QLEDs by methylamine intercalation. Small, 2019, 15: 1902735

31 Wu G, Zheng X, Cui P, et al. A general synthesis approach for amorphous noble metal nanosheets. Nat Commun, 2019, 10: 4855

32 Wang $\mathrm{S}, \mathrm{Bi} \mathrm{C}$, Yuan J, et al. Original core-shell structure of cubic CsPbBr ${ }_{3} @$ amorphous $\mathrm{CsPbBr}_{x}$ perovskite quantum dots with a high blue photoluminescence quantum yield of over $80 \%$. ACS Energy Lett, 2018, 3: 245-251

33 Liu J, Zheng M, Shi X, et al. Amorphous FeOOH quantum dots assembled mesoporous film anchored on graphene nanosheets with superior electrochemical performance for supercapacitors. Adv Funct Mater, 2016, 26: 919-930

34 Wang Q, Liu Y, Chen P. Phenazine-based organic cathode for aqueous zinc secondary batteries. J Power Sources, 2020, 468:
228401

35 Liang Q, Li Z, Huang $\mathrm{ZH}$, et al. Holey graphitic carbon nitride nanosheets with carbon vacancies for highly improved photocatalytic hydrogen production. Adv Funct Mater, 2015, 25: 68856892

36 Li Y, Jin R, Xing Y, et al. Macroscopic foam-like holey ultrathin g$\mathrm{C}_{3} \mathrm{~N}_{4}$ nanosheets for drastic improvement of visible-light photocatalytic activity. Adv Energy Mater, 2016, 6: 1601273

37 Han Q, Wang B, Gao J, et al. Atomically thin mesoporous nanomesh of graphitic $\mathrm{C}_{3} \mathrm{~N}_{4}$ for high-efficiency photocatalytic hydrogen evolution. ACS Nano, 2016, 10: 2745-2751

$38 \mathrm{Yu} \mathrm{H}, \mathrm{Xu} \mathrm{J}$, Gao D, et al. Triethanolamine-mediated photodeposition formation of amorphous Ni-P alloy for improved $\mathrm{H}_{2}$ evolution activity of $\mathrm{g}-\mathrm{C}_{3} \mathrm{~N}_{4}$. Sci China Mater, 2020, 63: 2215-2227

39 Kang Y, Yang Y, Yin LC, et al. Selective breaking of hydrogen bonds of layered carbon nitride for visible light photocatalysis. Adv Mater, 2016, 28: 6471-6477

40 Yu H, Shi R, Zhao Y, et al. Alkali-assisted synthesis of nitrogen deficient graphitic carbon nitride with tunable band structures for efficient visible-light-driven hydrogen evolution. Adv Mater, 2017, 29: 1605148

41 Su FY, Xu CQ, Yu YX, et al. Carbon self-doping induced activation of $\mathrm{n}-\pi^{*}$ electronic transitions of $\mathrm{g}-\mathrm{C}_{3} \mathrm{~N}_{4}$ nanosheets for efficient photocatalytic $\mathrm{H}_{2}$ evolution. ChemCatChem, 2016, 8: 3527-3535

42 Yu S, Li J, Zhang Y, et al. Local spatial charge separation and proton activation induced by surface hydroxylation promoting photocatalytic hydrogen evolution of polymeric carbon nitride. Nano Energy, 2018, 50: 383-392

43 Liu Y, Duan W, Song W, et al. Red emission B, N, S-co-doped carbon dots for colorimetric and fluorescent dual mode detection of $\mathrm{Fe}^{3+}$ ions in complex biological fluids and living cells. ACS Appl Mater Interfaces, 2017, 9: 12663-12672

44 Shen P, Xia Y. Synthesis-modification integration: One-step fabrication of boronic acid functionalized carbon dots for fluorescent blood sugar sensing. Anal Chem, 2014, 86: 5323-5329

45 Wang Y, Li H, Yao J, et al. Synthesis of boron doped polymeric carbon nitride solids and their use as metal-free catalysts for aliphatic C-H bond oxidation. Chem Sci, 2011, 2: 446-450

46 Guo Q, Zhang Y, Qiu J, et al. Engineering the electronic structure and optical properties of g- $\mathrm{C}_{3} \mathrm{~N}_{4}$ by non-metal ion doping. J Mater Chem C, 2016, 4: 6839-6847

47 Xing W, Li C, Chen G, et al. Incorporating a novel metal-free interlayer into g- $\mathrm{C}_{3} \mathrm{~N}_{4}$ framework for efficiency enhanced photocatalytic $\mathrm{H}_{2}$ evolution activity. Appl Catal B-Environ, 2017, 203: 65-71

48 Zhao D, Dong CL, Wang B, et al. Synergy of dopants and defects in graphitic carbon nitride with exceptionally modulated band structures for efficient photocatalytic oxygen evolution. Adv Mater, 2019, 31: 1903545

49 Kawaguchi M, Kawashima T, Nakajima T. Syntheses and structures of new graphite-like materials of composition $\mathrm{BCN}(\mathrm{H})$ and $\mathrm{BC}_{3} \mathrm{~N}(\mathrm{H})$. Chem Mater, 1996, 8: 1197-1201

50 Zhang S, Li J, Zeng M, et al. Polymer nanodots of graphitic carbon nitride as effective fluorescent probes for the detection of $\mathrm{Fe}^{3+}$ and $\mathrm{Cu}^{2+}$ ions. Nanoscale, 2014, 6: 4157-4162

51 Li B, Peng W, Zhang J, et al. High-throughput one-photon excitation pathway in $0 \mathrm{D} / 3 \mathrm{D}$ heterojunctions for visible-light driven hydrogen evolution. Adv Funct Mater, 2021, 31: 2100816

52 Kang X, Kang Y, Hong X, et al. Improving the photocatalytic activity of graphitic carbon nitride by thermal treatment in a high- 
pressure hydrogen atmosphere. Prog Nat Sci-Mater Int, 2018, 28: 183-188

53 Lin Z, Wang X. Nanostructure engineering and doping of conjugated carbon nitride semiconductors for hydrogen photosynthesis. Angew Chem Int Ed, 2013, 52: 1735-1738

54 Guo S, Deng Z, Li M, et al. Phosphorus-doped carbon nitride tubes with a layered micro-nanostructure for enhanced visible-light photocatalytic hydrogen evolution. Angew Chem Int Ed, 2016, 55: 1830-1834

55 Patir K, Gogoi SK. Facile synthesis of photoluminescent graphitic carbon nitride quantum dots for $\mathrm{Hg}^{2+}$ detection and room temperature phosphorescence. ACS Sustain Chem Eng, 2018, 6: 17321743

56 Härd T, Fan P, Kearns DR. A fluorescence study of the binding of Hoechst 33258 and DAPI to halogenated DNAs. Photochem Photobiol, 1990, 51: 77-86

57 Loh KP, Bao Q, Eda G, et al. Graphene oxide as a chemically tunable platform for optical applications. Nat Chem, 2010, 2: 1015-1024

58 Li LL, Ji J, Fei R, et al. A facile microwave avenue to electrochemiluminescent two-color graphene quantum dots. Adv Funct Mater, 2012, 22: 2971-2979

59 Zhang Q, Sun X, Ruan H, et al. Production of yellow-emitting carbon quantum dots from fullerene carbon soot. Sci China Mater, 2017, 60: 141-150

60 Hao JN, Yan B. A water-stable lanthanide-functionalized MOF as a highly selective and sensitive fluorescent probe for $\mathrm{Cd}^{2+}$. Chem Commun, 2015, 51: 7737-7740

61 Lu M, Deng Y, Luo Y, et al. Graphene aerogel-metal-organic framework-based electrochemical method for simultaneous detection of multiple heavy-metal ions. Anal Chem, 2019, 91: 888895

62 Noh Y, Jo EJ, Mun H, et al. Homogeneous and selective detection of cadmium ions by forming fluorescent cadmium-protein nanoclusters. Chemosphere, 2017, 174: 524-530

63 Wang R, Wang R, Ju D, et al. "ON-OFF-ON" fluorescent probes based on nitrogen-doped carbon dots for hypochlorite and bisulfite detection in living cells. Analyst, 2018, 143: 5834-5840

64 de Silva AP, Gunaratne HQN, Gunnlaugsson T, et al. Signaling recognition events with fluorescent sensors and switches. Chem Rev, 1997, 97: 1515-1566

65 Barman S, Sadhukhan M. Facile bulk production of highly blue fluorescent graphitic carbon nitride quantum dots and their application as highly selective and sensitive sensors for the detection of mercuric and iodide ions in aqueous media. J Mater Chem, 2012, 22: 21832-21837

66 Wesp EF, Brode WR. The absorption spectra of ferric compounds. I. The ferric chloride-phenol reaction. J Am Chem Soc, 1934, 56: 1037-1042

Acknowledgements This work was supported by the National Natural Science Foundation of China (51772085 and 12072110) and the Natural Science Foundation of Hunan Province (2020JJ4190).

Author contributions $\quad \mathrm{Li}$ B, Huang WQ, Hu W and Huang GF conceptualized this research. Li B carried out the preparation and tests of the composites. Zhang J, Luo Z, Duan X, Pan A, Liao L and Jiang L contributed to the X-ray photoelectron spectroscopy, atomic force microscopy and time-resolved fluorescence lifetime analyses. Li B, Huang WQ, $\mathrm{Hu} \mathrm{W}$, and Huang GF co-wrote the paper. All of the authors contributed to the general discussion.

Conflict of interest The authors declare no conflict of interest.

Supplementary information Supporting data are available in the online version of this paper.

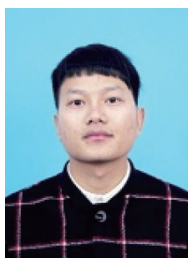

Bo Li received his bachelor's degree from Shangqiu Normal University in 2016. He is currently a PhD candidate at the College of Materials Science and Engineering, Hunan University. His current research interests include controllable growth and physical properties of nanomaterials and catalysis for energy and environmental research.

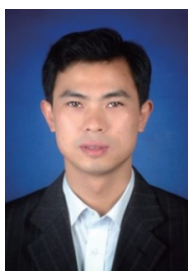

Wei-Qing Huang received his $\mathrm{PhD}$ degree from Hunan University in 2005. Following his stint as a postdoctoral fellow with Prof. Baiyun Huang at the Central South University and as a visiting scholar with Prof. Baowen Li at the National University of Singapore, he now works as a full professor at the College of Physics and Electronics, Hunan University. His current research interests focus on condensed matter physics and functional nanomaterials in energy-related areas.

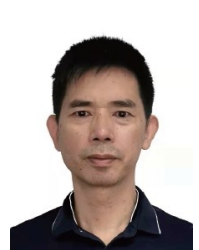

Wangyu $\mathrm{Hu}$ is a professor at Hunan University. He obtained his $\mathrm{PhD}$ degree in materials science from the Institute of Metals, Chinese Academy of Sciences. In 1996, he moved to the State Key Laboratory of Powder Metallurgy, Central South University, as a postdoctoral fellow. In 1999, he attended the University of Liverpool in the UK with the support of the Royal Society. His research is committed to the development of the embedded atom model for use in the analyses of nanostructures, alloy design, irradiation damage, surface interface, and material properties under extreme high-temperature and high-pressure conditions.

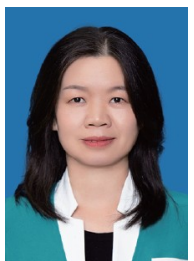

Gui-Fang Huang is a professor at the College of Physics and Electronics, Hunan University. She received her bachelor's degree from the Department of Chemistry and Chemical Engineering in 1996 and her $\mathrm{PhD}$ degree from the College of Materials Science and Engineering, Hunan University, in 2005. Her current research interests include the controllable growth and physical properties of nanomaterials and catalysis for energy and environmental research. 


\section{非晶态硼掺杂石墨相氮化碳量子点: 近 $90 \%$ 高苂光 量子产率及高灵敏检测 $\mathrm{Fe}^{2+} / \mathrm{Cd}^{2+}$}

李波 ${ }^{1}$, 张静 ${ }^{2}$, 骆子显 ${ }^{1}, \mathbf{E}$ 金沛 ${ }^{3}$, 黄维清 ${ }^{1^{*}}$, 胡望宇 ${ }^{1^{*}}$, 潘安练 ${ }^{1}$, 廖蕾 $^{3}$, 江浪 $^{2}$, 黄桂芳 $^{1^{*}}$

摘要 石墨相氮化碳量子点(CNQDs)作为一种具有吸引力的光致 发光 (PL) 材料, 在苂光成像和重金属离子检测方面具有巨大的潜 力. 然而, 晶态CNQDs的商业化应用仍被其低苂光量子产率、自 猝灭和激发波长依赖行为所限制. 我们提出利用嗍掺杂和CNQDs 非晶化的协同策略一一通过水热和自上而下的超声剥离方法制备 非晶态嗍掺杂CNQDs (B-CNQDs)-—以解决以上三个问题. 结构 无序赋予了非晶态B-CNQDs大范围 $\mathrm{pH}$ 下优越的弹性应变性能, 从 而可以有效促进电荷传输, 减少激子猝灭. 同时, 通过形成均匀的硼 掺杂表面态, 可以巧妙地调节CNQDs的电子结构和苂光发射特性, 实现无激发波长依赖行为. 结果表明, 非晶态 $\mathrm{B}-\mathrm{CNQDs}$ 具有优异的 荧光稳定性 (2年后无明显衰减), 且具有 $87.4 \%$ 的高量子产率, 是非 常有前途的苂光材料. 在紫外光激发下, B-CNQDs在商业用纸上可 以显示出特定图形的亮蓝色荧光. 此外, B-CNQDs对 $\mathrm{Fe}^{2+}$ 和 $\mathrm{Cd}^{2+}$ 在 $\mathrm{ppb}$ 检测范围内具有高度的选择性和灵敏度. 本工作可为开发具有 高稳定性和高量子产率的非晶态碳基荧光材料用于光电器件和传 感器提供实验基础和有益启示. 